\title{
Relationship of Working Memory, Processing Speed, and Fluid Reasoning in Psychiatric Patients
}

\author{
Se-Jin Kim ${ }^{1}$ and Eun Hee Park ${ }^{1,2} \bowtie$ \\ ${ }^{1}$ Department of Psychiatry, Hallym University Sacred Heart Hospital, Hallym University College of Medicine, Anyang, Republic of Korea \\ ${ }^{2}$ Department of Psychology, Seoul National University, Seoul, Republic of Korea
}

Objective The present study aimed to investigate relationship among cognitive factors (working memory and processing speed) and fluid reasoning (Gf) in psychiatric patients using a standardized clinical tool.

Methods We included the responses of 115 heterogeneous patients who were diagnosed with the MINI-Plus 5.0 and WAIS-IV/WMSIV was administered. For our analysis, structured equation modeling (SEM) was conducted to evaluate which cognitive variables are closely related to the Gf.

Results The results showed that the visual working memory was the strongest predictor of the Gf compared to other cognitive factors. Conclusion Processing speed was capable of predicting the Gf, when visual working memory was controlled. The inter-relationship among the Gf and other cognitive factors and its clinical implications were further discussed. Psychiatry Investig 2018;15(12):1154-1161

Key Words Working memory, Processing speed, Fluid reasoning, WAIS-IV, WMS-IV.

\section{INTRODUCTION}

According to the theory of fluid and crystallized intelligences, initially developed by Cattell, fluid intelligence or fluid reasoning (Gf) is 'a capacity to think logically and solve problems in novel situations. ${ }^{1}$ Since working memory and processing speed have been proposed as primary cognitive factors of the Gf, many theoretical works were introduced in context of cognitive development, education, learning, and assessment in general. ${ }^{2,3}$ Recently, understanding the interrelationship among these cognitive abilities have been considered important for interpreting results from neuropsychological evaluations in clinical settings; however, very few clinical studies have been recognized. Therefore, the present study aims to investigate the relationship among the primary

Received: July 6, 2018 Revised: September 5, 2018

Accepted: October 10, 2018

$\triangle$ Correspondence: Eun-Hee Park, MA

Department of Psychiatry, Hallym University Sacred Heart Hospital, Hallym University College of Medicine, 22 Gwanpyeong-ro 170beon-gil, Dongan-gu, Anyang 14068, Republic of Korea

Department of Psychology, Seoul National University, 1 Gwanak-ro, Gwanakgu, Seoul 08826, Republic of Korea

Tel: +82-31-380-3752, Fax: +82-31-381-3751, E-mail: psypeh@hanmail.net

(c) This is an Open Access article distributed under the terms of the Creative Commons Attribution Non-Commercial License (http://creativecommons.org/licenses/bync/4.0) which permits unrestricted non-commercial use, distribution, and reproduction in any medium, provided the original work is properly cited. cognitive factors (working memory and processing speed) and Gf in a clinical sample using a standardized clinical tool.

Working memory is one of the core executive functions that acts as a bridge between short-term and long-term memory by holding and manipulating input information. ${ }^{4}$ Many researchers used to conceive working memory as a single process, ${ }^{5}$ but recent studies have re-defined working memory to be a multiple process. Baddeley and Hitch later theorized working memory consists of domain-general storage (the central executive), responsible for incorporating all the information, and domain-specific storage (the phonological loop and visuospatial sketchpad) which separated from one another and work independently. ${ }^{6,7}$ According to this model, the phonological loop processes auditory information while the visuospatial sketchpad deals with visual/spatial information. Furthermore, different matters such as the type of stimuli, level of task difficulty, and modality rationale, affect the activation of different parts of the working memory system. ${ }^{8,9}$ This signifies the necessity to take both perspectives of the domain-specific (stands out when specific component of the stimuli take over the priority of the relationship) and domain-general (stands out when active manipulation of information is necessary) into account when dealing with working memory. Majority of working memory span tasks were developed based on Baddeley and Hitch's theory of working 
memory and it is continuously being used in the present. ${ }^{10}$

Processing speed, first introduced as a 'performance speed', was differentiated according to its content by Marshalek et al. ${ }^{11}$ Since then, the concept of processing speed became more complex and its definition started to become re-defined by various researchers. ${ }^{12,13}$ Processing speed is now considered as a multi-dimensional factor that is representative of one's cognitive performance in a designated amount of time. ${ }^{14}$ and it is also highly related to working memory and Gf. Ackerman et al. ${ }^{15}$ conducted confirmatory factor analysis (CFA) of the content ability factors to equate constructs of working memory within processing speed. According to their findings, processing speed is sub-divided into four underlying factors: processing speed-complex, -memory, -pattern recognition, and -scanning ability. Out of the four factors, processing speed-memory, which represents incidental memory and associated learning, was highly correlated with working memory.

Even though such studies have suggested the possible link between working memory and processing speed, limited study has been done to clarify their relationship. Oberauer et al. ${ }^{16}$ showed some communality between working memory and mental speed when one engages in tasks with low-level complexity. Some early longitudinal studies have successfully addressed that working memory and processing speed both are related with the Gf. ${ }^{14,17,18}$ Recent findings have shown working memory has a stronger relationship with Gf than with the processing speed. For instance, Conway et al. ${ }^{19}$ conducted a latent variable analysis of the Gf and other cognitive factors (working memory, processing speed, and short-term memory), and their results showed the working memory was the only variable qualified to predict the Gf. Furthermore, recent findings from neuroimaging studies consolidated the relationship between the Gf and working memory by elucidating their neural substrates, ${ }^{20}$ the direct/indirect relationship between white matter integrity and the Gf through working memory. ${ }^{21}$

Previous literature succeeded to identify the crucial roles of the working memory and processing speed when explaining the Gf. However, the relationship among these three factors in the clinical population is yet to be undisclosed. Thus, the present research aims to investigate which of the two cognitive factors (working memory and processing speed) would be the better indicator of Gf in psychiatric patients using standardized tools (e.g., Wechsler Adult Intelligence Scale-IV; WAIS-IV and Wechsler Memory Scale-IV; WMSIV). ${ }^{22,23}$ WAIS-IV and WMS-IV were chosen for not only its high accessibility as the measurement of cognitive function in clinical settings, but also because of its norm-referenced format. Based on previous literatures, we hypothesized that the working memory would be the better predictor of the Gf than the processing speed.

\section{METHODS}

\section{Participants}

We included the responses of 115 heterogeneous patients with ages from 19 to 60, all diagnosed with either Schizophrenia, Bipolar Disorder, Depressive Disorder, Anxiety Disorder, Head Trauma due to Traumatic Brain Injury, Mental Retardation, or Autism. The diagnosis were made according to the criteria of the Diagnostic and Statistical Manual of Mental Disorder fourth edition (DSM-IV) ${ }^{24}$ and the Korean version of MINI International Neuropsychiatric Interview Plus Version 5.0 (K-MINI-Plus 5.0). ${ }^{25}$ All the patients have been asked for their informed consent prior to the studies, and further inclusion screening was preceded to only those who agreed to participate in the study. Exclusion criteria were of the following: 1) patients with hearing, vision, or fine motor deficits and 2) patients with other medical conditions (e.g., chronic psychosomatic, cardiovascular, or respiratory diseases). Diagnostically, the sample consisted of $13.19 \%$ patients with schizophrenia, $19.27 \%$ with major depressive disorder, 3.40\% with bipolar disorder, $15.21 \%$ with anxiety disorder, $9.13 \%$ with developmental disorder, $8.12 \%$ with neurocognitive disorder, and 3.40\% with other psychiatric conditions. The mean and standard deviation of gender, level of education, age, and sample size among each clinical group are provided in Table 1.

\section{Materials}

Based on Baddeley's theoretical works, we sub-divided working memory into two components (visual/auditory) and we postulated four latent variables; eight observed variables were selected from Korean Wechsler Adult Intelligence ScaleIV (K-WAIS-IV) ${ }^{26}$ and Korean Wechsler Memory Scale-IV (K-WMS-IV). ${ }^{27}$ Each observed variables represented the target latent variables of: 1) digit span/letter number sequencing represent the auditory working memory, 2) spatial addition/ symbol span represent the visual working memory, 3) metrics reasoning/figure weight represent the Gf, and 4) symbol search/coding represent processing speed. Figure 1 illustrates the basis of the model structure for our analysis. The circles represent latent constructs, and the squares represent the observed variables.

WAIS-IV is designed to assess one's intellectual abilities with four cognitive domains (verbal comprehension, perceptual reasoning, working memory, and processing speed) based on numerous statistically validated studies and theoretical foundations. They are composed of 15 subtests that well 
Table 1. Demographic analysis

\begin{tabular}{|c|c|c|c|}
\hline & $\begin{array}{l}\text { Gender } \\
\text { (F\%) }\end{array}$ & Education & Age \\
\hline \multicolumn{4}{|c|}{ Schizophrenia $(\mathrm{N}=18)$} \\
\hline Mean & 40.0 & 12.667 & 30.733 \\
\hline SD & & 2.160 & 12.331 \\
\hline \multicolumn{4}{|c|}{ Major depressive disorder $(\mathrm{N}=39)$} \\
\hline Mean & 33.3 & 12.970 & 27.455 \\
\hline SD & & 2.129 & 11.025 \\
\hline \multicolumn{4}{|c|}{ Bipolar disorder $(\mathrm{N}=6)$} \\
\hline Mean & 25.0 & 11.750 & 30.750 \\
\hline $\mathrm{SD}$ & & 6.344 & 16.879 \\
\hline \multicolumn{4}{|c|}{ Anxiety disorder ( $\mathrm{N}=24)$} \\
\hline Mean & 30.4 & 14.304 & 32.826 \\
\hline $\mathrm{SD}$ & & 2.382 & 14.389 \\
\hline \multicolumn{4}{|c|}{$\begin{array}{l}\text { Developmental disorder }(\mathrm{N}=13) \\
\text { (mental retardation, autism) }\end{array}$} \\
\hline Mean & 41.7 & 10.333 & 29.000 \\
\hline SD & & 3.393 & 12.247 \\
\hline \multicolumn{4}{|c|}{ Neurocognitive disorder $(\mathrm{N}=10)$} \\
\hline Mean & 22.2 & 11.333 & 45.556 \\
\hline SD & & 5.172 & 16.432 \\
\hline \multicolumn{4}{|c|}{ Others $(\mathrm{N}=5)$} \\
\hline Mean & 20.0 & 13.400 & 23.400 \\
\hline $\mathrm{SD}$ & & 1.949 & 4.615 \\
\hline \multicolumn{4}{|c|}{ Total $(\mathrm{N}=115)$} \\
\hline Mean & 32.7 & 12.743 & 30.891 \\
\hline SD & & 3.104 & 13.438 \\
\hline
\end{tabular}

SD: standard deviation

represent each of their domains. ${ }^{22}$ In a similar context with WAIS-IV, WMS-IV is also a statistically validated and theorybased clinical tool, composed of subtests that well represent each of their domains as well. ${ }^{28,29}$ WMS-IV was developed to evaluate various memory functions such as working memory, immediate and delayed recall, and recognition. ${ }^{23}$

\section{Fluid reasoning}

Fluid Reasoning, known as the Gf, represents the mental manipulation skills such as abstract symbol reasoning. ${ }^{23}$ In this study, the Gf was measured using two subtests from WAIS-IV: Matrix Reasoning and Figure Weight. Matrix Reasoning measures the non-verbal perceptual reasoning abilities including the fluid reasoning ability, which has been included since WAIS-III. Wechsler has added Figure Weight as a supplementary subtest to emphasize the importance of the Gf within the perceptual reasoning index.

\section{Auditory working memory}

Digit Span and Letter-Number Sequencing were selected, as for the observed variables for measuring the auditory working memory. The two subtests (Digit Span, LetterNumber Sequencing) measure auditory processing speed, auditory attention span, working memory, and mental manipulation. ${ }^{30}$ The WAIS-IV strengthens the working memory load in Digit Span subtest by adding a sequencing trial. ${ }^{22}$ Letter-Number Sequencing has recently been recognized as the best clinical working memory task. ${ }^{14,26,31,32}$

\section{Visual working memory}

For this study, we only utilized subtests which were designed to measure the visual working memory (Spatial Addition and Symbol Span). Spatial Addition measures the mental manipulation of spatial information, working memory, and attention, while Symbol Span measures the working memory for abstract visual materials. ${ }^{33}$

\section{Processing speed}

To measure the processing speed, we included Symbol Search and Coding subtests in the WAIS-IV as our observed variables. Both subtests require adequate visuomotor processing speed, visual scanning, attention, and short-term memory. According to Ackerman et al., ${ }^{15}$ scanning ability accounts for a big portion of the Symbol Search subtest, while the memory component plays a more essential role in the Coding subtest.

\section{Procedure}

Prior to conducting the research, all procedures were approved by Hallym University Sacred Heart Hospital Institutional Review Board (IRB No. 2015-I097). Participants were tested individually by clinical psychology trainees, under the supervision of a qualified clinical psychologist. All the assessments were administrated in a single session of approximately an hour, during which pre-selected subtests from WAISIV were conducted, followed by the visual working memory subtests from WMS-IV.

\section{Analyses}

The expectation-maximization algorithm ${ }^{34}$ was conducted to replace the missing values. The confirmatory factor analysis (CFA) was performed to verify the model fit for the measurement model. The structured equation modeling (SEM) was conducted to evaluate which latent variables were closely related to the Gf. Several fit indices were used to evaluate the fit of each model to the data. The $\mathrm{p}$ value associated with below 0.05 was considered an acceptable model fit. However, since the sample size is influential in chi-square statistic, ${ }^{35}$ additional fit 
Table 2. Correlations among all tasks

\begin{tabular}{|c|c|c|c|c|c|c|c|c|}
\hline \multirow{2}{*}{ Task } & \multicolumn{2}{|c|}{ Auditory working memory } & \multicolumn{2}{|c|}{ Processing speed } & \multicolumn{2}{|c|}{ Visual working memory } & \multicolumn{2}{|c|}{ Fluid reasoning(Gf) } \\
\hline & DS & $\mathrm{LN}$ & SS & $\mathrm{CD}$ & SA & SSP & MR & FW \\
\hline DS & 1 & & & & & & & \\
\hline $\mathrm{LN}$ & $0.853^{*}$ & 1 & & & & & & \\
\hline SS & $0.639 *$ & $0.610^{*}$ & 1 & & & & & \\
\hline $\mathrm{CD}$ & $0.705^{*}$ & $0.659^{*}$ & $0.829^{*}$ & 1 & & & & \\
\hline SA & $0.530^{*}$ & $0.466^{*}$ & $0.519^{*}$ & $0.485^{*}$ & 1 & & & \\
\hline SSP & $0.563^{*}$ & $0.568^{*}$ & $0.602^{*}$ & $0.590^{*}$ & $0.686^{*}$ & 1 & & \\
\hline MR & $0.685^{*}$ & $0.637^{*}$ & $0.737^{*}$ & $0.745^{*}$ & $0.620^{*}$ & $0.634^{*}$ & 1 & \\
\hline FW & $0.637^{*}$ & $0.631^{*}$ & $0.679^{*}$ & $0.679^{*}$ & $0.697^{*}$ & $0.689^{*}$ & $0.797^{*}$ & 1 \\
\hline
\end{tabular}

*significant at $\mathrm{p}<0.01$. DS: Digit Span, LN: Letter Number Sequencing, SA: Spatial Addition, SSP: Symbol Span, SS: Symbol Search, CD: Coding, MR: Matrix Reasoning, FW: Figure Weight

measures were utilized; the root mean squared error of approximation (RMSEA; cut off $<0.06$ ), ${ }^{36}$ Tucker-Lewis nonnormed fit index (TLI; cut off $>0.95$ ), ${ }^{37}$ comparative fit index (CFI; cut off $>0.90$ ), ${ }^{36}$ and the goodness-of-fit index (GFI; 1996; cut off $>0.95)^{38}$ were also used to evaluate the model fit. Furthermore, Fisher's $\mathrm{z}$ transformation was performed to assess the significance of the difference between the correlation coefficients among working memory, processing speed, and the Gf. The raw scores were used in all statistically analysis and were carried by SPSS version 20 and AMOS version 22 .

\section{RESULTS}

\section{Descriptive statistics}

Correlations among all the cognitive tasks are presented in Table 2 , which clearly shows positive inter-correlations among the 8 tasks (range $=0.466-0.853$ ).

\section{Model fit for measurement model}

The CFA was performed to verify the model fit for the measurement model (Figure 1). The model fit the data well for the measurement model $\left(\chi^{2}=18.493, \mathrm{df}=14, \mathrm{p}=0.151\right.$, RM$\mathrm{SEA}=0.019$, TLI $=0.990, \mathrm{CFI}=0.995$, and $\mathrm{GFI}=0.960$ ). Each of the indicators was well loaded on its respective latent constructs. Factor loadings ranged between $0.79-0.95$ and all the paths from the observed variable to the latent constructs were significant at 0.05 . Additionally, the path coefficient of Gf and each of the latent variables are the following: visual working memory (path coefficient $=0.95, \mathrm{p}<0.05$ ), auditory working memory (path coefficient $=0.80, \mathrm{p}<0.05$ ), and processing speed (path coefficient $=0.88, \mathrm{p}<0.05$ ).

\section{Association among the three cognitive factors and the Gf}

To verify the relationship among the three cognitive factors,

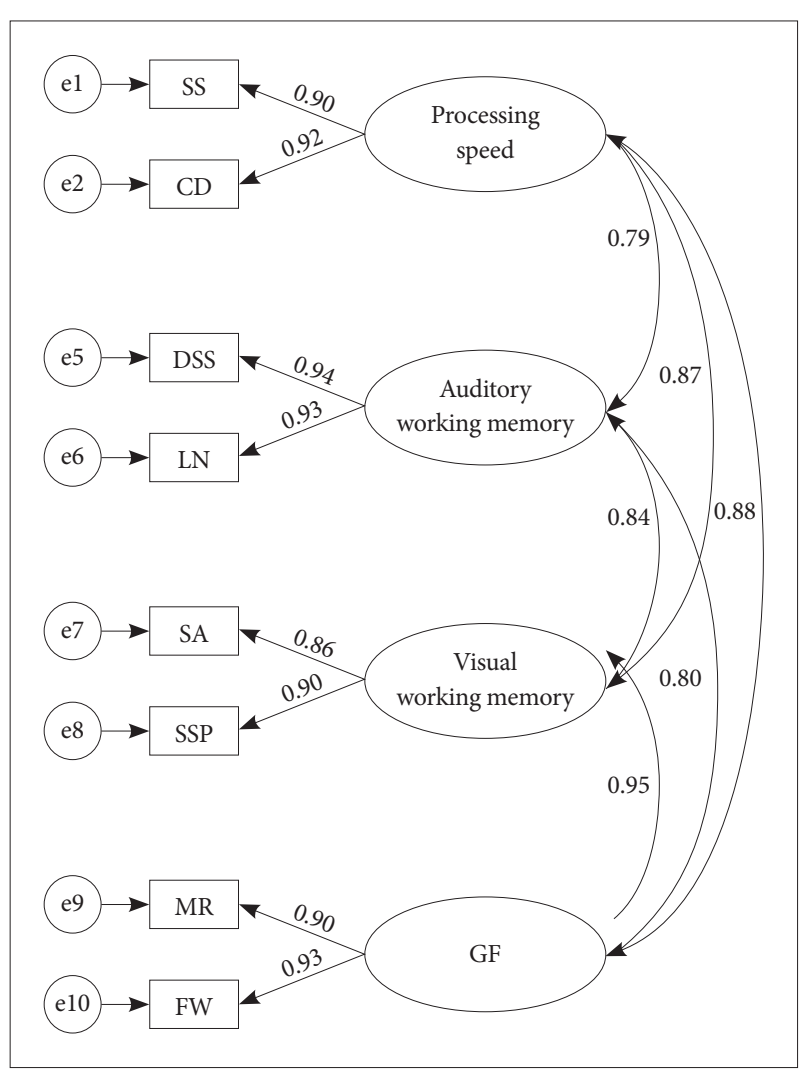

Figure 1. Measurement model. The circles represent latent constructs, and the squares represent the observed variables. Single-headed arrows represent standardized factor loadings and double-headed arrows represent correlations between factors. All parameter estimates are standardized and significant at $p<0.05$. DS: Digit Span, LN: Letter Number Sequencing, SA: Spatial Addition, SSP: Symbol Span, SS: Symbol Search, CD: Coding, MR: Matrix Reasoning, FW: Figure Weight.

processing speed, visual/auditory working memory, and the Gf, SEM was performed (Table 3). Overall model fit for the model was good $\left(\chi^{2}=19.386, \mathrm{df}=14, \mathrm{p}=0.151, \mathrm{RMSEA}=0.019\right.$, $\mathrm{TLI}=0.998, \mathrm{CFI}=0.994$, and $\mathrm{GFI}=0.957)$. It was found that only visual working memory was able to predict the Gf (path 
coefficient $=0.78, \mathrm{p}<0.01$ ) (Figure 2), out of the factors.

Furthermore, we observed how much of these constructs explained the variance of Gf to evaluate how visual/auditory working memory and processing speed are related with the Gf. This was executed by removing the processing speed, then the visual and auditory working memory paths one at a time from the unconstrained model. In the first model, path from the auditory working memory to the Gf was constrained to zero. Model fit was adequate and it was not significantly different to the unconstrained model $\left(\chi^{2}=19.409, \mathrm{df}=14\right.$, $\mathrm{p}=0.196$, RMSEA $=0.019$, TLI $=0.991, \mathrm{CFI}=0.995$, and $\mathrm{GFI}=0.957 ; \chi_{\text {diff }}^{2}=0.084, \mathrm{df}_{\text {diff }}=1, \mathrm{p}=0.772$ ).

In our second model, path from the visual working memory to the Gf was also constrained to zero. When compared to the unconstrained model, a significant change was found. Once we removed the visual working memory from the model, the fit became slightly worse compared to the uncon- strained model $\left(\chi^{2}=38.366, \mathrm{df}=14, \mathrm{p}=0.001, \mathrm{RMSEA}=0.031\right.$, $\mathrm{TLI}=0.953, \mathrm{CFI}=0.990$, and $\mathrm{GFI}=0.920 ; \chi_{\text {diff }}^{2}=18.483, \mathrm{df}_{\text {diff }}=1$, $\mathrm{p}<0.010$ ). Furthermore, once visual working memory was under control, processing speed was able to predict the Gf (path coefficient $=0.78, \mathrm{p}<0.050$ ).

In the third model, path from the processing speed to the Gf was constrained to zero as well. There was no significant difference when compared to the unconstrained model $\left(\chi^{2}=21.104, \mathrm{df}=14, \mathrm{p}=0.134, \mathrm{RMSEA}=0.021, \mathrm{TLI}=0.998\right.$, $\mathrm{CFI}=0.993$, and $\mathrm{GFI}=0.955 ; \chi_{\text {diff }}^{2}=1.611, \mathrm{df}_{\text {diff }}=1, \mathrm{p}=0.204$ ), which signified only the visual working memory was able to predict the Gf, out of the three.

For further analysis, using the Fisher's $\mathrm{z}$ transformation, ${ }^{39}$ we calculated a value of $\mathrm{z}$ that can be applied to assess the significance of the difference between the correlation coefficients of working memory \& Gf and processing speed \& Gf. The results showed the difference between $\mathrm{A}_{\left(\mathrm{z}^{\prime} \text { for visual working }\right.}$

Table 3. Goodness-of-fit for SEM

\begin{tabular}{|c|c|c|c|c|c|c|c|c|}
\hline \multirow{2}{*}{ Model } & \multicolumn{6}{|c|}{ Goodness-of-fit indices } & \multicolumn{2}{|c|}{ Model comparison } \\
\hline & $\chi^{2}$ & $\mathrm{p}$ & RMSEA & TLI & CFI & GFI & $\chi_{\text {diff }}^{2}$ & $\mathrm{p}$ \\
\hline Measurement model & 18.493 & 0.151 & 0.019 & 0.990 & 0.995 & 0.960 & - & - \\
\hline Unconstrained model & 19.386 & 0.151 & 0.019 & 0.998 & 0.994 & 0.957 & - & - \\
\hline Model 1 (VWM+PS) & 19.409 & 0.196 & 0.019 & 0.991 & 0.995 & 0.957 & 0.084 & 0.772 \\
\hline Model 2 (AWM+PS) & 38.366 & 0.001 & 0.031 & 0.953 & 0.975 & 0.920 & 18.843 & $<0.01$ \\
\hline Model 3 (AWM+VWM) & 21.104 & 0.134 & 0.021 & 0.998 & 0.993 & 0.955 & 1.611 & 0.204 \\
\hline
\end{tabular}

SEM: Structured Equation Modeling, RMSEA: Root Mean Squared Error of Approximation, TLI: Tucker-Lewis Nonnormed Fit Index, CFI: Comparative Fit Index, GFI: Goodness-of-Fit Index, $\chi_{\text {diff }}^{2} \chi^{2}$ difference, AWM: auditory working memory, VWM: visual working memory, PS: processing speed

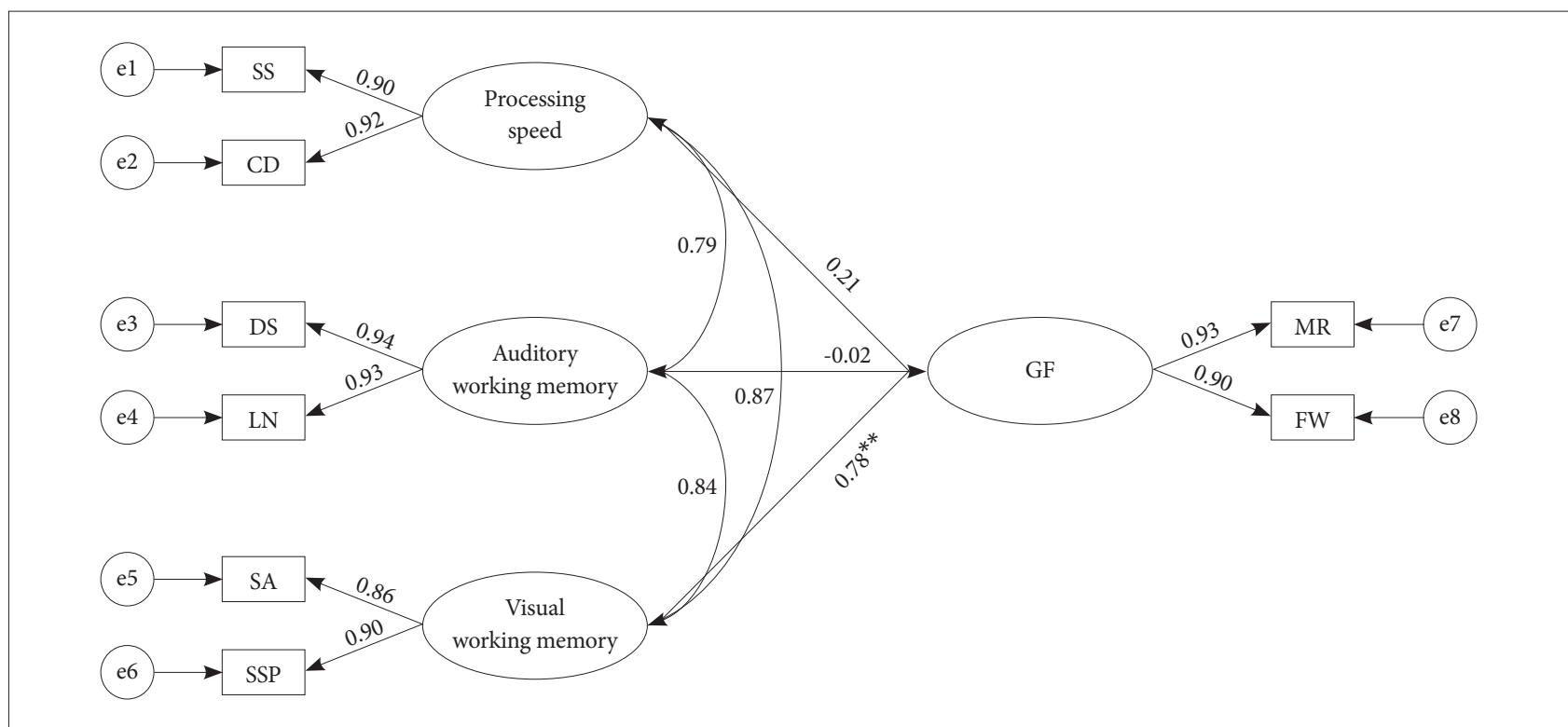

Figure 2. Unconstrained model. The circles represent latent constructs, and the squares represent the observed variables. Single-headed arrows represent standardized factor loadings and double-headed arrows represent correlations between factors. All parameter estimates are standardized and significant at ${ }^{* *} p<0.01$. DS: Digit Span, LN: Letter Number Sequencing, SA: Spatial Addition, SSP: Symbol Span, SS: Symbol Search, CD: Coding, MR: Matrix Reasoning, FW: Figure Weight. 
memory\&the Gf) Vs. $\mathrm{B}_{(\mathrm{z}}$ for auditory working memory\&the Gf) $(\mathrm{z}=5.487, \mathrm{p}<0.010)$ and $\mathrm{A}_{(\mathrm{z}}$ ' for visual working memory\&the Gf) Vs. $\mathrm{C}_{(\mathrm{z}}$ ' for processing speed\&the Gf) $(\mathrm{z}=3.412, \mathrm{p}<0.010)$ were statistically significant. Also, there was statistically significant difference $B_{(z}$ for auditory working memory \& the Gf) Vs. $C_{(z}$ for processing speed \& the Gf) $(z=-2.074, p=0.038)$. In summary, visual working memory showed stronger relationship with the Gf than with the auditory working memory and processing speed.

\section{DISCUSSION}

Working memory and processing speed were known to be the critical cognitive factors of explaining the Gf for several decades. As far as we know, most of the previous researches were based on normal samples, and studies involving clinical populations are rare. And studies utilizing standardized psychological assessments with such populations are even more uncommon. Our study was designed to understand the Gf within the critical cognitive factors (working memory and processing speed) in a mixed clinical sample by utilizing standardized tools that are widely used over the world. We hypothesized the working memory would be the better predictor of the Gf compared to the processing speed, and our results partially confirm this hypothesis in that the visual working memory appears to be the better predictor of the Gf than the processing speed. Processing speed was able to predict the Gf only when visual working memory was controlled. The present study attempts to illustrate the relationship amongst cognitive factors (working memory and processing speed) and Gf in psychiatric patients using standardized clinical tools with a goal to allow clinicians and researchers to generate further clinical interpretations. Our results have clinicians to keep in mind that other cognitive variables that are inter-related with the Gf should be account on its interpretations in clinical setting.

When it comes to comparing working memory and processing speed, working memory takes all the credit for predicting the Gf; this result was predictable in two ways. According to the classic model from Baddley, working memory could be represented as either domain-general or domainspecific. Shipstead and Yonehiro ${ }^{40}$ suggested the domain-general characteristic of working memory stood out when active manipulation of information was necessary. On the other hand, if the content of task was visuospatially skewed, domain-specific component would take over the priority of the relationship. For example, in Shah and Miyake's study, ${ }^{41}$ verbal working memory predicted the performance on the verbal reasoning task, while high level of spatial working memory was capable of predicting a good performance on the visuospatial reasoning task. The current study was only able to confirm the domain-specific relationship between the Gf and working memory. And the potential reasons are as follows: 1) sub-tests that were represented as the Gf in this study contain a visual modality component and 2) contrary to what the tasks were originally designed for, tasks for auditory working memory in WAIS-IV still include more of the domain-specific component. For instance, Egeland ${ }^{42}$ investigated the differences in complexity/manipulation load (processing model) and modality difference (modality model) among the working memory sub-tests in WAIS-IV by conducting the CFA. His study reported that the processing model was not strong enough to distinguish working memory elements, while the modality model successfully fit the data.

Kail and Salthouse ${ }^{17}$ addressed working memory was highly related to processing speed, and Fry and Hale ${ }^{43}$ concluded that the working memory, processing speed, and the Gf took a similar trajectory over time. These results suggest the three variables are highly influential to one another and developmentally in concert. In the present study, processing speed was capable of predicting the Gf after the visual working memory was controlled for. This result may be understood as two reasons as follows: 1) communality between working memory and processing speed and 2) time limit component in subtests of observed variable for Gf. There is communality between working memory and processing speed. In other words, because the two factors are closely related, working memory may overshadow the processing speed as the predictor of the Gf. On the other perspective, processing speed carries the ability to process information quickly; thus time limit component in observed variables of Gf, especially the Figure Weight, may influence the relationship between the processing speed and the Gf.

This study shows strengths in various ways. First, the study is based on clinical cases, which is an approach that have not been tried and applied in previous researches. Understanding inter-relationship among various cognitive abilities is important in clinical setting because a cognitive deficit revealed from neuropsychological assessment could be resulted by various dysfunction of its underlying cognitive skills. For example, a patient who shows deficit in the Gf on her neuropsychological assessment can be interpreted in one of the two ways: 1) low capacity of the Gf and 2) low capacity (or deficit) in underlying cognitive factors of the Gf such as working memory. Gf is a complex and higher-order cognitive function requiring several basic cognitive abilities (e.g., working memory or processing speed) to be able to exert its ability. ${ }^{44}$ In other words, deficit in one of the lower-level cognitive abilities such as working memory can cause the decline in Gf. Thus, interpreting the Gf in a context of lower-order cognitive factors would be necessary in clinical settings. In this context, 
even though it is not recommended for all the patients, ${ }^{33}$ conducting visual working memory sub-tests from the WMS-IV may enrich the clinicians' clinical interpretation.

Second, standardized clinical assessments were used to explain the relationships between the Gf and other cognitive abilities. Taking a different route from the previous approaches, WAIS-IV uses four first-order factor index scores (verbal comprehension, perceptual reasoning, working memory and processing speed) based on the theoretical foundations. It allows clinicians to see the relationship between Gf and two crucial factors (working memory and processing speed) within a standardized manner. Many researches have been done in a laboratory setting. In most of these cases, researchers develop their own laboratory tasks suitable for measuring what they are looking for, because theoretical/underlying definition of each study varies depending on the study's purpose. One of the drawbacks from using these laboratory tasks is the absence of the standardised norms, which makes comparing the task results from other experiments is not favorable. Thus, the present study acquires credit for utilizing standardized clinical tools allowing for replication and comparison of its results.

Some considerations must be taken into account for future studies. The current study was administered in a single institution with a relatively small sample size, which limits the results from becoming generalized for all the population. The sample is also heterogeneous, which may cause the relationships between the variables be invariant across the different subgroups. Those with neurocognitive or developmental disorders are likely to have different parameter values than those with anxiety or mood disorders. Since the present study is a cross-sectional design, the results from this study cannot determine the causality among the three cognitive factors but only its correlation. However, despite the aforementioned limitations, this study revealed working memory as the strongest predictor of the Gf by utilizing standardized clinical tests. Although, profile/part score analyses have their limits as a diagnostic utility, these results will serve to help make further interpretive implications from the measurements of cognitive deficits in an individual level. As above reasons suggest, it would be necessary for future studies to explore the relationship between working memory and the Gf within specific clinical groups from multi-institutional settings and with a large sample size.

\section{REFERENCES}

1. Cattell RB. Theory of fluid and crystallized intelligence: a critical experiment. J Educ Psychol 1963;54:1-22.

2. Zeller F, Wang T, Reiss S, Schweizer K. Does the modality of measures influence the relationship among working memory, learning and fluid intelligence? Pers Individ Dif 2017;105:275-279.

3. Tourva A, Spanoudis G, Demetriou A. Cognitive correlates of devel- oping intelligence: The contribution of working memory, processing speed and attention. Intelligence 2016;54:136-146.

4. Baddeley AD, Hitch GJ. Developments in the concept of working memory. Neuropsychology 1994;8:485-493.

5. Miller GA. The magical number seven, plus or minus two: some limits on our capacity for processing information. Psychol Rev 1956;63:81-97.

6. Baddeley A. The episodic buffer: A new component of working memory? Trends Cogn Sci 2000;4:417-423.

7. Baddeley AD, Hitch G. Working memory. Psychol Learn Motiv 1974;8:47-89.

8. Just MA, Carpenter PA, Keller TA, Emery L, Zajac H, Thulborn KR. Interdependence of nonoverlapping cortical systems in dual cognitive tasks. Neuroimage 2001; 14: 417-426.

9. Sirevaag EJ, Kramer AF, Coles MGH, Donchin E. Resource reciprocity: an event-related brain potentials analysis. Acta Psychol (Amst) 1989;70:77-97.

10. Conway AR, Kane MJ, Bunting MF, Hambrick DZ, Wilhelm O, Engle RW. Working memory span tasks: A methodological review and user's guide. Psychon Bull Rev 2005;12:769-786.

11. Marshalek B, Lohman DF, Snow RE. The complexity continuum in the radex and hierarchical models of intelligence. Intelligence 1983;7:107-127.

12. Kyllonen PC, Christal RE. Reasoning ability is (little more than) working-memory capacity?! Intelligence 1990;14:389-433.

13. Kyllonen PC, Tirre WC, Christal RE. Knowledge and processing speed as determinants of associative learning. J Exp Psychol Gen 1991;120: 57-79.

14. Salthouse TA. The processing-speed theory of adult age differences in cognition. Psychol Rev 1996;103:403-428.

15. Ackerman PL, Beier ME, Boyle MD. Individual differences in working memory within a nomological network of cognitive and perceptual speed abilities. J Exp Psychol Gen 2002;131:567-589.

16. Oberauer K, Suss M, Schulze R, Wilhelm O, Wittmann WW. Working memory capacity-facets of a cognitive ability construct. Pers Individ Dif 2000;29:1017-1045.

17. Kail R, Salthouse TA. Processing speed as a mental capacity. Acta Psychol 1994;86:199-225.

18. Verhaeghen P, Salthouse TA. Meta-analyses of age-cognition relations in adulthood: Estimates of linear and nonlinear age effects and structural models. Psychol Bull 1997;122:231-249.

19. Conway ARA, Cowan N, Bunting MF, Therriault DJ, Minkoff SRB. A latent variable analysis of working memory capacity, short-term memory capacity, processing speed, and general fluid intelligence. Intelligence 2002;30:163-183.

20. Barbey AK, Colom R, Paul EJ, Grafman J. Architecture of fluid intelligence and working memory revealed by lesion mapping. Brain Struct Funct 2014;219:485-494.

21. Privado J, Sáenz de Urturi C, Dávila J, López C, Burgaleta M, Román FJ, et al. White matter integrity predicts individual differences in (fluid) intelligence through working memory. Pers Individ Dif 2014;60:S77.

22. Wechsler D, Coalson DL, Raiford SE. WAIS-IV. San Antonio, TX: Pearson; 2008.

23. Wechsler D. Wechsler Memory Scale: WMS-IV: Technical and Interpretive Manual. San Antonio, TX: Pearson; 2009.

24. APA. Diagnostic and Statistical Manual of Mental Disorders-IV-TR. Washington, DC: American Psychiatric Association; 2000.

25. Yoo S, Kim Y, Noh J, Oh K, Kim C, Oh D. Validity of Korean version of the mini-international neuropsychiatric interview. Anxiety Mood 2006;2:5-50.

26. Hwang S, Kim J, Park K, Chey J, Hong S. Korean Wechsler Adult Intelligence Test (4th Ed.). Daegu: Korean Psychol; 2011.

27. Chey J, Kim J, Park G, Hong S, Hwang S, Shin M. Standardization of the K-WMS-IV. Korean Psychol Assoc 2012;1.

28. Hoelzle JB, Nelson NW, Smith CA. Comparison of Wechsler Memory Scale-Fourth Edition (WMS-IV) and Third Edition (WMS-III) dimensional structures: Improved ability to evaluate auditory and visual 
constructs. J Clin Exp Neuropsychol 2011;33:283-291.

29. Holdnack JA, Zhou X, Larrabee GJ, Millis SR, Salthouse TA. Confirmatory factor analysis of the WAIS-IV/WMS-IV. Assessment 2011;18: 178-191.

30. Reynolds CR. Forward and backward memory span should not be combined for clinical analysis. Arch Clin Neuropsychol 1997;12:29-40.

31. Shelton JT, Elliott EM, Hill BD, Calamia MR, Gouvier WD. A comparison of laboratory and clinical working memory tests and their prediction of fluid intelligence. Intelligence 2009;37:283-293.

32. Crowe SF. Does the letter number sequencing task measure anything more than digit span? Assessment 2000;7:113-117.

33. Weiss LG, Saklofske DH, Coalson D, Raiford SE. WAIS-IV Clinical Use and Interpretation: Scientist-Practitioner Perspectives. London, UK: Academic Press; 2010.

34. Dempster AP, Laird NM, Rubin DB. Maximum likelihood from incomplete data via the EM algorithm. J R Stat Soc 1977;1:1-38.

35. Schumacker RE, Lomax RG. A Beginner's Guide to Structural Equation Modeling. Abingdon-on-Thames: Routledge; 2012.

36. Bentler PM. Comparative fit indexes in structural models. Psychol Bull 1990;107:238-246.

37. Tucker LR, Lewis C. A reliability coefficient for maximum likelihood factor analysis. Psychometrika 1973;38:1-10

38. Baumgartner H, Homburg C. Applications of structural equation modeling in marketing and consumer research: a review. Int J Res Mark 1996;13:139-161.

39. Cohen P, West SG, Aiken LS. Applied multiple regression/correlation analysis for the behavioral sciences. Brighton, UK: Psychology Press; 2014.

40. Shipstead Z, Yonehiro J. The domain-specific and domain-general relationships of visuospatial working memory to reasoning ability. Psychon Bull Rev 2016;23:1504-1512.

41. Shah P, Miyake A. The separability of working memory resources for spatial thinking and language processing: an individual differences approach. J Exp Psychol Gen 1996;125:4-27.

42. Egeland J. Measuring working memory with Digit Span and the Letter-Number Sequencing subtests from the WAIS-IV: too low manipulation load and risk for underestimating modality effects. Appl Neuropsychol Adult 2015;22:445-451.

43. Fry AF, Hale S. Processing speed, working memory, and fluid intelligence: Evidence for a developmental cascade. Psychol Sci 1996;7:237-241.

44. Hebben N, Milberg W. Essentials of Neuropsychological Assessment. Hoboken, NJ: John Wiley\&Sons; 2009. 